\title{
Subnormal Peripheral Blood Leukocyte Counts Are Related to the Lowest Prevalence and Incidence of Metabolic Syndrome: Tianjin Chronic Low-Grade Systemic Inflammation and Health Cohort Study
}

\author{
Shaomei Sun, ${ }^{1}$ Hongmei Wu, ${ }^{2}$ Qing Zhang, ${ }^{1}$ Chongjin Wang, ${ }^{1}$ Yinting Guo, \\ Huanmin Du, ${ }^{2}$ Li Liu, ${ }^{1}$ Qiyu Jia, ${ }^{1}$ Xing Wang, ${ }^{1}$ Kun Song, ${ }^{1}$ and Kaijun Niu, \\ ${ }^{1}$ Health Management Centre, Tianjin Medical University General Hospital, Tianjin, China \\ ${ }^{2}$ Nutritional Epidemiology Institute and School of Public Health, Tianjin Medical University, \\ 22 Qixiangtai Road, Heping District, Tianjin 300070, China \\ Correspondence should be addressed to Kaijun Niu; nkj0809@gmail.com
}

Received 21 November 2013; Accepted 2 April 2014; Published 29 April 2014

Academic Editor: Dianne Cooper

Copyright (C) 2014 Shaomei Sun et al. This is an open access article distributed under the Creative Commons Attribution License, which permits unrestricted use, distribution, and reproduction in any medium, provided the original work is properly cited.

Few studies have assessed the relationship between a subnormal inflammatory status and metabolic syndrome (MS). We therefore designed a cross-sectional and 5-year cohort study to evaluate how a subnormal peripheral blood leukocyte count is related to MS. Participants were recruited from Tianjin Medical University General Hospital-Health Management Centre. Both a baseline crosssectional $(n=46,179)$ and a prospective assessment $(n=13,061)$ were performed. Participants without a history of MS were followed up for 5 years. Leukocyte counts and MS components were assessed at baseline and yearly during the follow-up. Adjusted logistic and Cox proportional hazards regression models were used to assess relationships between the categories of leukocyte counts and MS. The subnormal leukocyte counts group $\left(1,100-3,900\right.$ cells $\left./ \mathrm{mm}^{3}\right)$ had the lowest prevalence and incidence of MS. The odds ratio and hazard ratio (95\% confidence interval) of the highest leukocyte counts were 1.98 (1.57-2.49) and 1.50 (1.22-1.84) (both $P$ for trend $<0.0001)$, respectively, when compared to the subnormal leukocyte counts group after adjusting for potential confounders. This study has shown that subnormal leukocyte counts are independently related to the lowest prevalence and incidence of MS. The findings suggest that it is necessary to restudy and discuss the clinical or preventive value of subnormal leukocyte counts.

\section{Introduction}

Chronic diseases, such as cardiovascular diseases (CVD), cancer, and age-related diseases, have long been considered among the most important global public health issues [1]. Clarifying the common pathological process of these diseases or statuses is a crucial step toward providing early prevention and treatment. A growing body of evidence indicates that chronic low-grade inflammation is a common pathological process and an important contributing factor to these diseases or statuses [2-4].

Various inflammatory markers, including soluble adhesion molecules (e.g., E-selectin, P-selectin, intracellular adhesion molecule-1, and vascular cell adhesion molecule-1), cytokines (e.g., interleukin- $1 \beta,-6,-8$, and -10 and tumor necrosis factor- $\alpha$ ), acute phase reactants (e.g., fibrinogen, serum amyloid A protein, and high sensitive C-reactive protein [hsCRP]), and leukocyte counts, are frequently used to assess systemic inflammatory statuses [2]. Among these inflammatory markers, peripheral blood leukocyte counts are routinely measured in clinical practice and are the only cellular marker of systemic inflammation. Furthermore, compared to other inflammatory markers, only leukocyte counts were given a lower limiting value: $<4,000$ cells $/ \mathrm{mm}^{3}$. However, it is noteworthy that this reference value is based only on the $95 \%$ lower limit in the general population [5].

To date, several cross-sectional [6-8] and cohort [9-11] studies have found that leukocyte counts are positively related 
to the prevalence and incidence of metabolic syndrome (MS), which is a well-recognized risk factor for CVD. However, most of these studies have used quartile or quintile categories of leukocyte counts to assess the relationship. Because the percentage of participants who have subnormal leukocyte counts is generally less than $8 \%$ among the general population, these studies cannot accurately evaluate the relationship between subnormal leukocyte counts and MS among an apparently healthy population. Further, one cohort study limited leukocyte counts from 4,000 to 10,000 cells $/ \mathrm{mm}^{3}$ during assessment [12]. It is, therefore, still unclear how subnormal leukocyte counts are related to MS.

Considering the above factors, we designed a crosssectional and 5-year follow-up study to investigate how a subnormal leukocyte count is related to the prevalence and incidence of MS in apparently healthy adults.

\section{Methods}

2.1. Participants. Tianjin chronic low-grade systemic inflammation and health (TCLSIH) cohort study is a large prospective dynamic cohort study focusing on the relationships between chronic low-grade systemic inflammation and the health status of a population living in Tianjin, China [13]. Tianjin is a city of approximately 10.43 million inhabitants, located in the northeast of the North China Plain, facing the Bohai Sea [14]. Participants were recruited, while having a routine annual physical examination in 2007 at Tianjin Medical University General Hospital-Health Management Centre, the largest and most comprehensive physical examination center in Tianjin.

The TCLIH data from 2007 to 2012 was used in this study. The participant selection process is described in Figure 1. During the research period, there were 49,872 participants who had received at least one health examination, agreed to participate, and provided informed consent for their data to be analyzed. We excluded participants who did not have leukocyte counts ( $n=497$ ) or body height and/or body weight measurements $(n=50)$ or those with a history of CVD $(n=2,774)$ or cancer $(n=372)$. Owing to these exclusions, the final cross-sectional study population comprised 46,179 participants (mean standard deviation age: 45.112 .5 years; male: 59.6\%).

For follow-up analysis, participants were excluded at baseline if they had received a health examination only in $2012(n=21,550)$ or had MS $(n=6,250)$. 5,318 participants who did not undergo health examinations during follow-up were also excluded. Following these exclusions, the final cohort study population comprised 13,061 participants (follow-up rate: 71.1\%; mean standard deviation age: 43.512 .2 years; male: $57.2 \%$ ). The protocol of our study was approved by the Institutional Review Board of the Tianjin Medical University. This study conforms to STROBE guidelines for cross-sectional and cohort studies.

2.2. Assessment of Leukocyte Counts. Fasting blood samples were taken by venipuncture of the cubital vein and immediately mixed with EDTA. Leukocyte and its differential counts were carried out using the automated hematology analyzer XE-2100 (Sysmex, Kobe, Japan) and expressed as $\times 1,000$ cells $/ \mathrm{mm}^{3}$. The test for blanks was $\leq 0.2 \times 10^{9}$ cells $/ \mathrm{L}$; the intra- and interassay coefficients of variation $(\mathrm{CV})$ were $\leq 2.0 \%$; and the cross-contamination rate was $\leq 0.5 \%$. In order to investigate how a subnormal leukocyte count is related to prevalence and incidence of MS, we divided participants into 8 categories according to leukocyte counts as follows: 1.1-3.9 (subnormal group), 4.0-4.9, 5.0-5.9, 6.0-6.9, 7.0-7.9, 8.0-8.9, 9.0-9.9, and $\geq 10.0$ (high-normal group) (cells $\left./ \mathrm{mm}^{3} \times 1,000\right)$. Furthermore, because neutrophils (50-60\%) and lymphocytes $(20-40 \%)$ constituted the predominant proportion of total circulating leukocytes, we also examine the relationships between quintiles of neutrophil and lymphocyte counts and MS.

2.3. Assessment of MS. Waist circumference was measured at the umbilical level with participants standing and breathing normally. Blood pressure (BP) was measured twice from the upper left arm using a TM-2655P automatic device (A\&D Co., Tokyo, Japan) after 5 minutes of rest in a seated position. The mean of these 2 measurements was taken as the BP value. Blood samples for the analysis of fasting blood sugar (FBS) and lipids were collected in siliconized vacuum plastic tubes. FBS was measured by the glucose oxidase method, triglycerides (TG) were measured by enzymatic methods, low-density lipoprotein cholesterol (LDL) was measured by the polyvinyl sulfuric acid precipitation method, and highdensity lipoprotein cholesterol (HDL) was measured by the chemical precipitation method using appropriate kits on a Cobas 8000 analyzer (Roche, Mannheim, Germany). Serum uric acid levels were determined according to a phosphotungstic acid reduction method with the Cobas 8000. Albumin in serum was measured by the bromocresol green method with the Cobas 8000. Plasma fibrinogen, which is a predictor of stroke and myocardial infarction [15], was determined by the freezing method with the autoanalyzer CA-1500 (Sysmex).

MS was defined in accordance with the criteria of the American Heart Association scientific statements of 2009 [16]. Participants were considered to have MS when they presented three or more of the following components.

(i) Elevated waist circumference for Chinese individuals ( $\geq 85 \mathrm{~cm}$ and $\geq 80 \mathrm{~cm}$ in women and men, resp.).

(ii) Elevated TG $(\geq 1.7 \mathrm{mmol} / \mathrm{L})$ or drug treatment for elevated TG.

(iii) Reduced HDL ( $<1.0 \mathrm{mmol} / \mathrm{L}$ in $\mathrm{men} ;<1.3 \mathrm{mmol} / \mathrm{L}$ in women) or drug treatment for reduced HDL.

(iv) Elevated blood pressure (SBP $\geq 130 \mathrm{~mm} \mathrm{Hg}$ and/or $\mathrm{DBP} \geq 85 \mathrm{~mm} \mathrm{Hg}$ ) or antihypertensive drug treatment.

(v) Elevated fasting glucose $(\geq 5.56 \mathrm{mmol} / \mathrm{L})$ or drug treatment of elevated glucose.

2.4. Assessment of Other Variables. Anthropometric parameters (height and body weight) were recorded using a standard protocol. Body mass index (BMI) was calculated as 


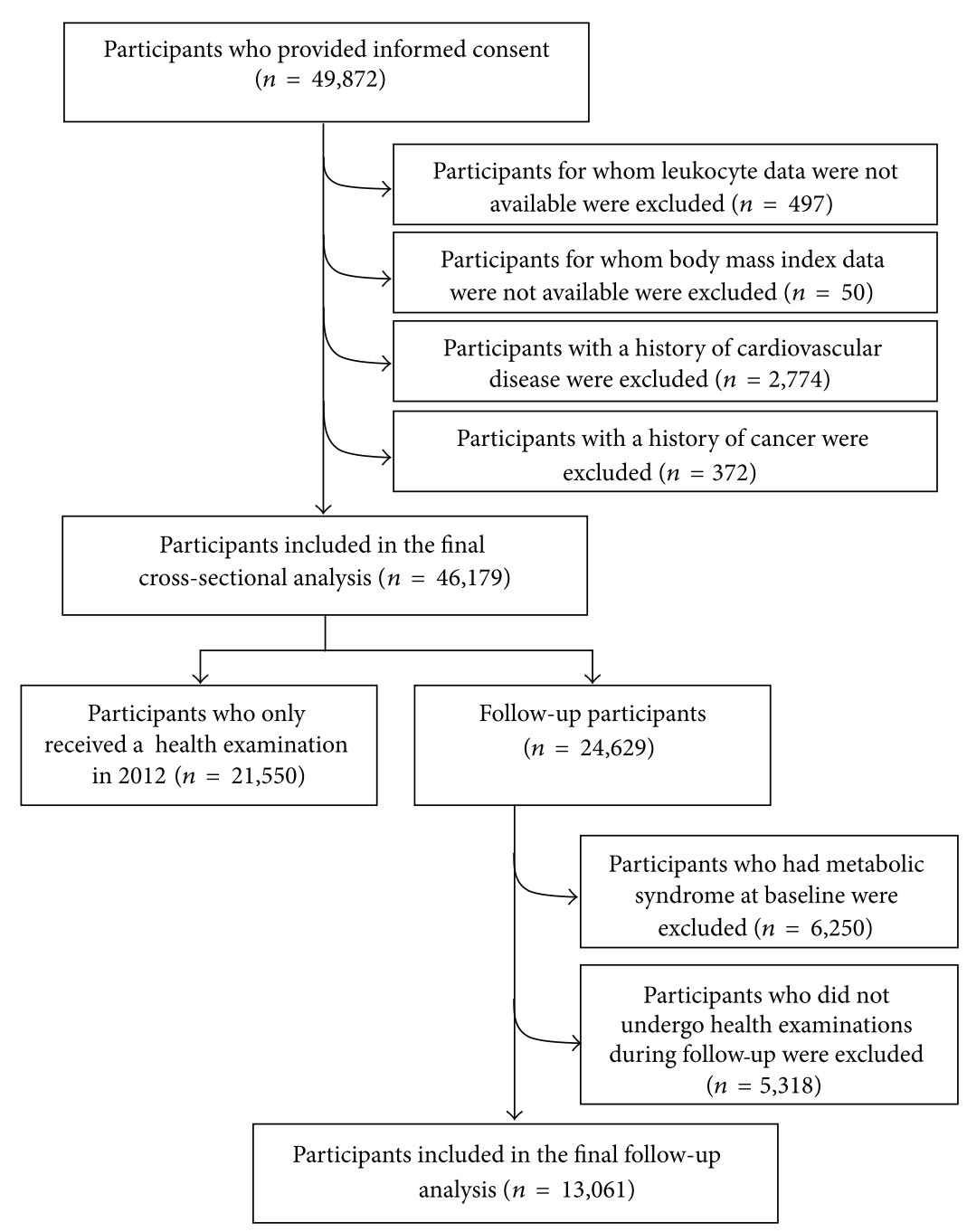

FIGURE 1: Selection of the study population, Tianjin chronic low-grade inflammation and health (TCLIH) cohort study, 2007 to 2012.

weight $/$ height ${ }^{2}\left(\mathrm{~kg} / \mathrm{m}^{2}\right)$. Sociodemographic variables, including gender, and age were also assessed. A detailed personal and family history of physical illness and current medications was noted from "yes" or "no" responses to relevant questions. Information on smoking and drinking statuses was obtained from a questionnaire survey.

2.5. Statistical Analysis. All statistical analyses were performed using the Statistical Analysis System version 9.3 for Windows (SAS Institute Inc., Cary, NC, USA). Descriptive data is presented as the mean (95\% confidence interval, CI) for adjusted continuous variables and as percentages for categorical variables.

For analysis, the prevalence and incidence of MS were used as dependent variables, and categories of leukocyte counts and quintiles of neutrophil and lymphocyte counts were used as independent variables. For baseline characteristics analysis, the differences among leukocyte count categories were examined using analysis of covariance (ANCOVA) for continuous variables and multiple logistic regression analysis for proportional variables after adjustment for age and sex. Multiple logistic regression analysis was used to examine relationships between leukocyte count categories, quintiles of neutrophil and lymphocyte counts, and the prevalence of MS after adjustment for covariates: age, sex, baseline BMI, smoking status, drinking status, and family history of CVD, hypertension, hyperlipidemia, and diabetes. The Cox proportional hazards regression model was used to examine the relationships between leukocyte count categories, quintiles of neutrophil and lymphocyte counts, and the incidence of MS with adjustment for the covariates mentioned above.

An odds ratio (OR), hazard ratio (HR), and 95\% CI were calculated. All $P$ values for linear trends were calculated using the median value of leukocyte count categories or quintiles of neutrophil and lymphocyte counts. All tests were two-tailed and $P<0.05$ was defined as statistically significant. 


\section{Results}

From 2007 to 2012, prevalence of MS was 19.8, 24.7, 28.8, 29.9, 33.4 , and 34.4, respectively. The percentages of participants with a subnormal leukocyte count were $7.7 \%$ and $8.6 \%$ for cross-sectional and follow-up analysis, respectively.

Age- and sex-adjusted participant characteristics in relation to leukocyte count categories for cross-sectional analysis are presented in Table 1. Compared to participants in the subnormal leukocyte counts group, those in the highest category tended to be younger and to have higher BMI, waist circumference, TC, TG, LDL, SBP, DBP, FBS, serum UA, and fibrinogen and lower HDL. A higher proportion of these participants were male, with a higher proportion of current smokers and alcohol consumers and a higher proportion of family history of hypertension and diabetes ( $P$ for all trends $\leq 0.02$ ). Other than these results, no significant differences were observed between participants in the leukocyte count categories.

The crude and adjusted relationships between leukocyte count categories and MS and its components are indicated in Table 2. In the final multivariate models, the adjusted ORs (95\% CI) of MS were related to the gradual increase of the categories of leukocyte counts as compared with participants who had subnormal leukocyte counts and were as follows: 1.19 (1.06, 1.34), 1.56 (1.39, 1.74), $1.74(1.55,1.96), 2.02$ (1.78, 2.29), 2.20 (1.90, 2.56), 2.28 (1.87, 2.77), and 1.98 (1.57, 2.49), respectively ( $P$ for trend $<0.0001)$. Similar relationships were also observed between the categories of leukocyte counts and MS components. Moreover, after adjustment for potential confounders, the ORs (95\% CI) of MS for increasing quintiles of neutrophil and lymphocyte counts were 1.00, 1.28 (1.18, 1.38), 1.44 (1.33, 1.56), $1.59(1.47,1.72)$, and $1.69(1.56,1.83)(P$ for trend <0.0001) and 1.00, $1.15(1.06,1.23), 1.32(1.20,1.45)$, $1.32(1.23,1.42)$, and $1.64(1.53,1.77)$ ( $P$ for trend $<0.0001)$, respectively (Figure $2(\mathrm{a})$ ).

Age- and sex-adjusted baseline characteristics for followup analysis are shown in Table 3. Because the number of participants in groups 6-8 of leukocyte counts was smaller and had essentially similar results, we combined these groups during subsequent statistical analysis. The baseline results were similar to the participant characteristics in cross-sectional analysis, with the exception of FBS and the proportion of those with a family history of hypertension. No significant differences were observed between the categories of leukocyte counts, FBS, and drinking status ( $P$ for trend $=0.72$ and 0.52 , resp.).

Incidence of MS was evaluated across the 5-year followup period. During this period, a total of 3,344 participants received a new diagnosis of MS. The incidence of MS was 113 per 1,000 person-years. Among the six leukocyte count groups, the respective rates of MS were $61,86,110,144$, 158 , and 160 per 1,000 person-years. The crude and adjusted relationships between categories of leukocyte counts and the incidence of MS are indicated in Table 4. In the crude model, the unadjusted HRs (95\% CI) of MS were related to the gradual increase of the categories of leukocyte counts as compared with participants who had subnormal leukocyte counts and were as follows: 1.40 (1.18, 1.66), 1.79 (1.51, 2.12), 2.34
$(1.97,2.77), 2.58(2.15,3.09)$, and $2.59(2.13,3.16)$, respectively ( $P$ for trend $<0.0001$ ). In the final multivariate models, the adjusted HRs (95\% CI) of MS were related to the gradual increase of the categories of leukocyte counts as compared with participants who had subnormal leukocyte counts and were as follows: $1.21(1.02,1.44), 1.35$ (1.14, 1.59), 1.58 (1.33, $1.88), 1.64(1.36,1.98)$, and $1.50(1.22,1.84)$, respectively $(P$ for trend $<0.0001)$. Moreover, after adjustment for potential confounders, the HRs (95\% CI) of MS for increasing quintiles of neutrophil and lymphocyte counts were 1.00, $1.22(1.08$, 1.37), 1.30 (1.16, 1.46), $1.29(1.15,1.44)$, and $1.41(1.25,1.58)(P$ for trend $<0.0001)$ and $1.00,1.12(1.01,1.25), 1.21(1.05,1.39)$, $1.22(1.10,1.35)$, and $1.30(1.16,1.44)$ ( $P$ for trend $<0.0001)$, respectively (Figure $2(\mathrm{~b})$ ).

\section{Discussion}

This study has examined the relationships between categories of leukocyte counts and MS in an apparently healthy population. Our results suggest that subnormal leukocytes counts were independently related to the lowest prevalence and incidence of MS. Similar relationships were also observed when neutrophil and lymphocyte counts were analyzed separately.

We adjusted for a number of potentially confounding factors in our analyses. Studies have shown that age and sex are simultaneously related to the incidence of MS and inflammatory status $[16,17]$; therefore, we first adjusted for age and sex. However, adjustment for these factors did not significantly affect the relationship between the categories of leukocyte counts and MS, leading us to believe that the direct relationship between leukocyte count categories and MS was independent of age and sex. We next adjusted for $\mathrm{BMI}$, which is an important risk factor which can cause a variety of adverse health effects [18]. Although the OR and HR for MS in higher leukocyte count categories, as compared with the subnormal leukocyte count groups, were greatly reduced, adjustment for this factor did not significantly affect the relationship between leukocyte count categories and MS. We subsequently adjusted for lifestyle factors including smoking (a major factor for leukocytes count increase [19, 20]), drinking status, and any effects of family history of diseases including CVD, hypertension, hyperlipidemia, and diabetes (which are recognized as genetic factors) of MS. However, these adjustments also did not affect the positive relationship between leukocyte count categories and MS.

Leukocyte counts are a simple, widely available, inexpensive, and well-standardized biomarker of inflammation. Several cross-sectional $[6-8,21,22]$ and cohort $[9-11,23,24]$ studies have suggested positive relationships between leukocyte counts and MS among populations in various countries both Asian and western. Furthermore, several cohort studies also suggest leukocyte counts as a potential predictor of allcause mortality and CVD mortality $[12,25]$. However, these studies have not definitively assessed the relationship between subnormal leukocyte counts and healthy states, therefore leaving the relationship unclear. A previous study of ours has shown that maintaining an inflammatory level (hsCRP was used as an inflammatory marker) as low as possible 


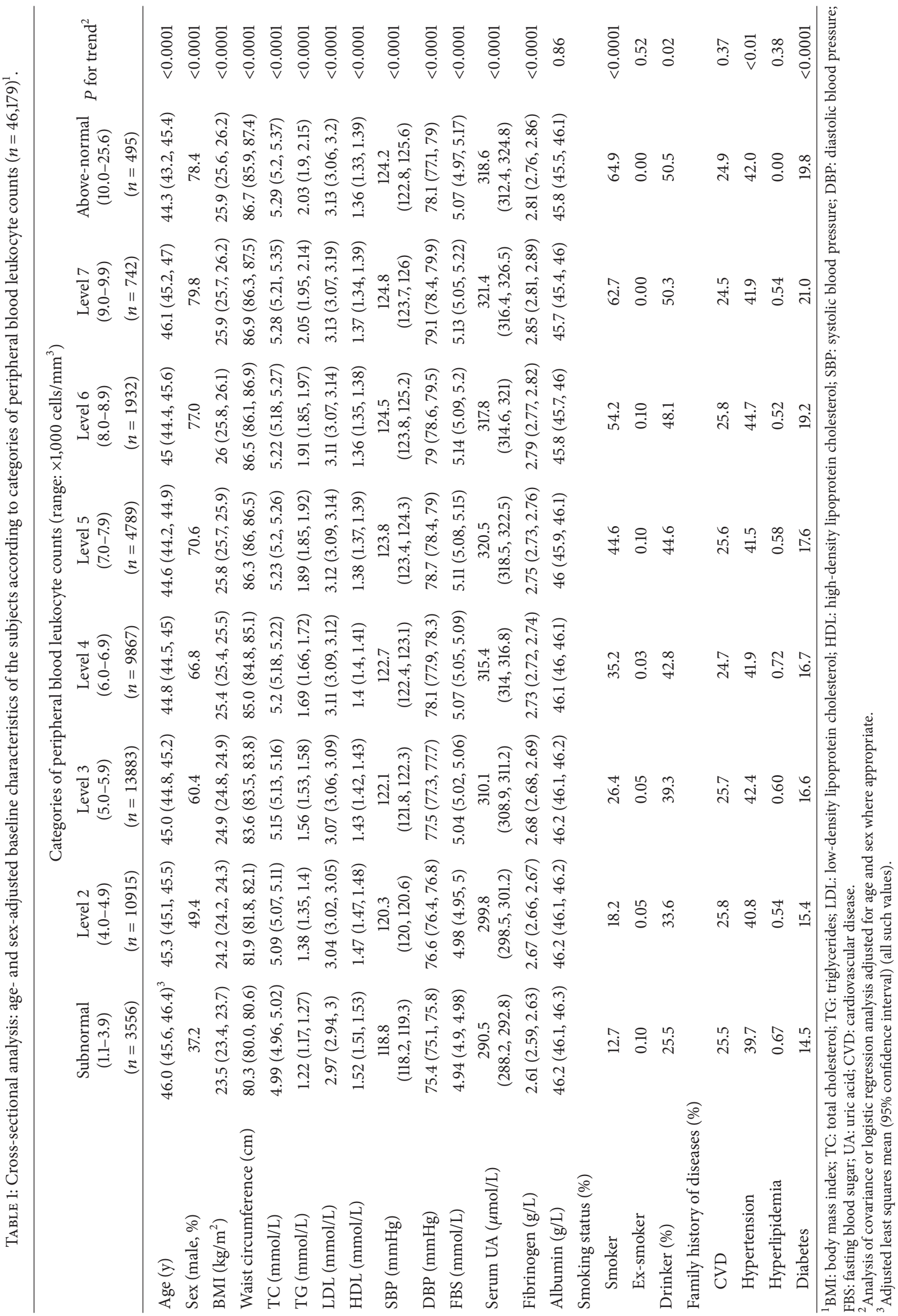




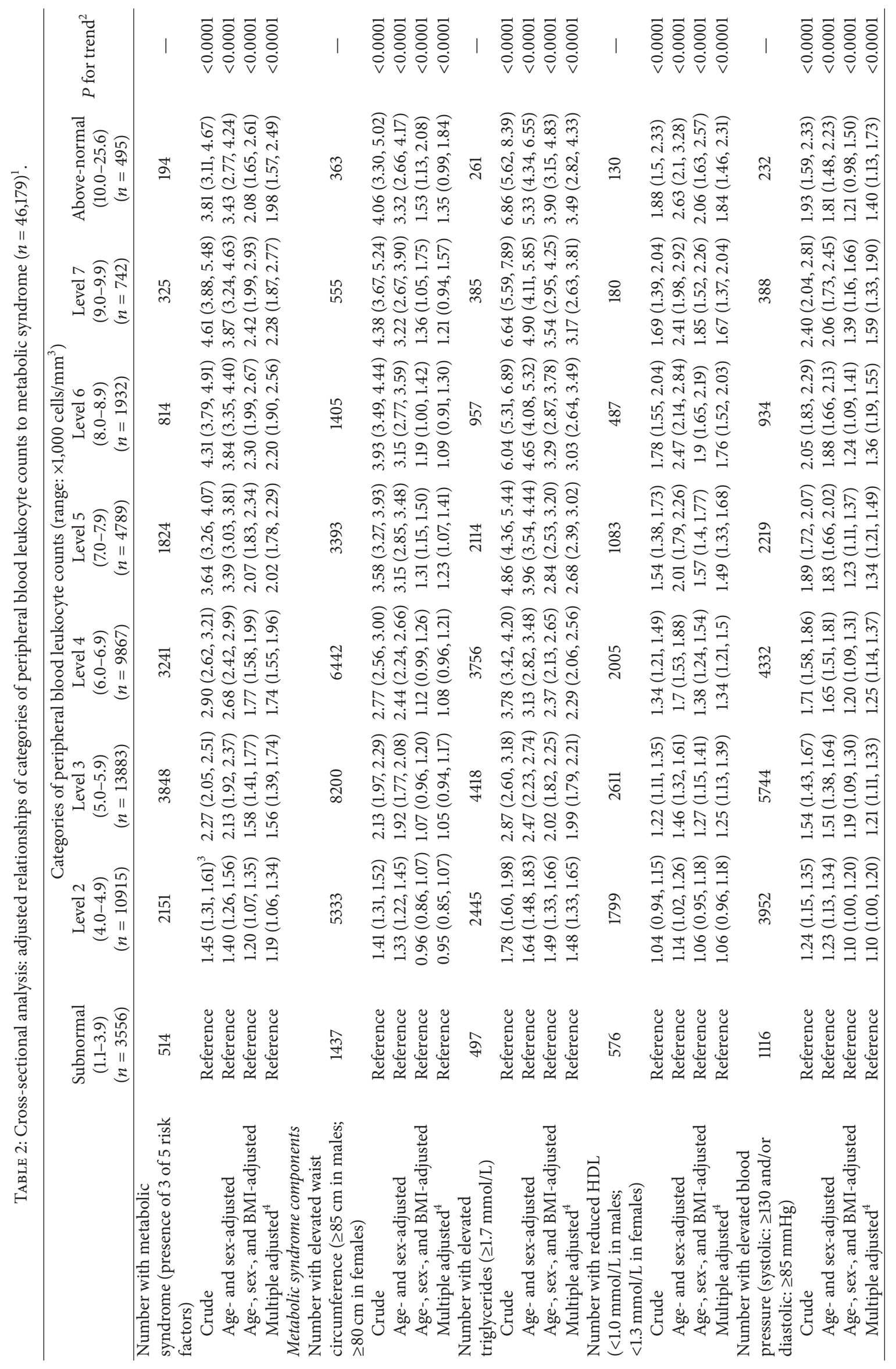




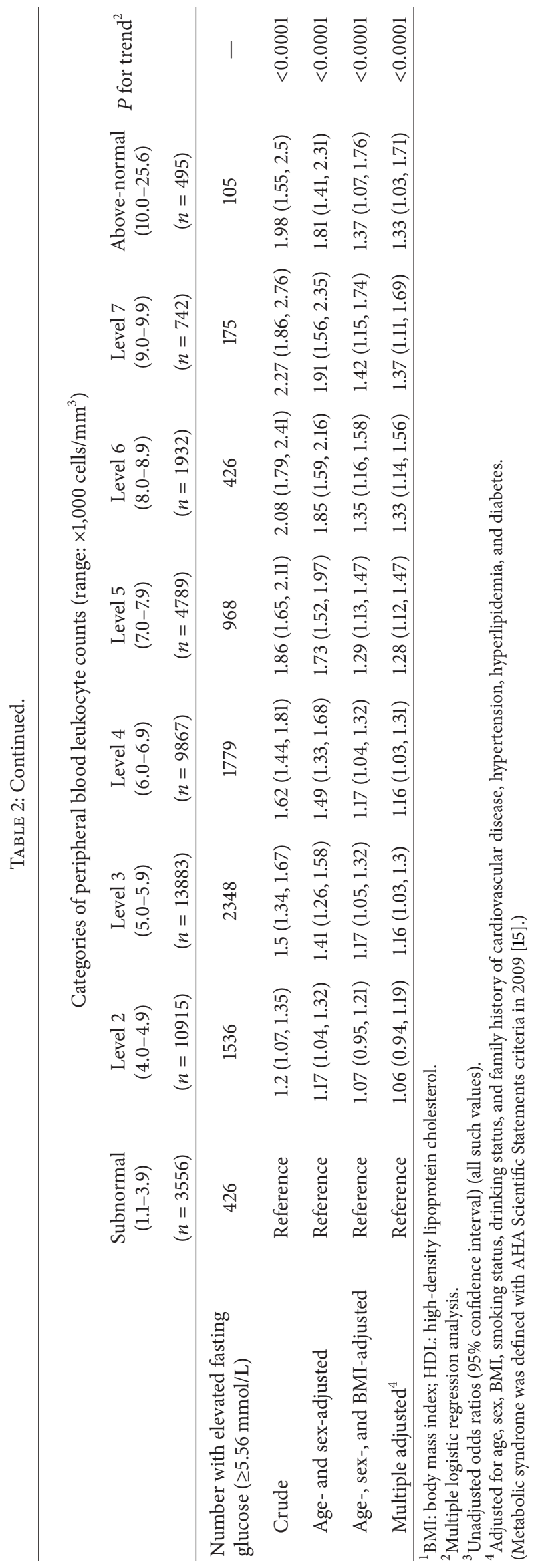




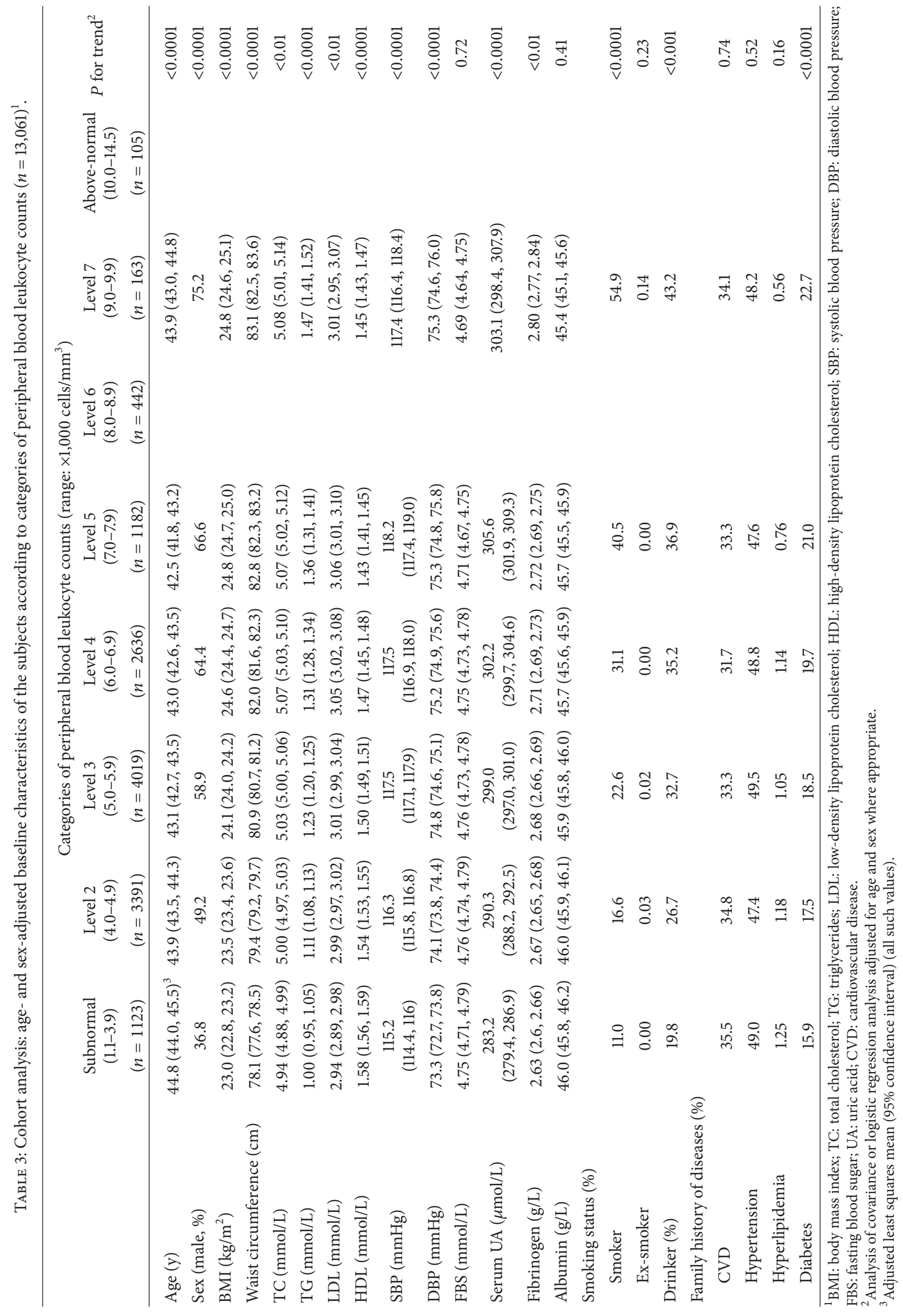




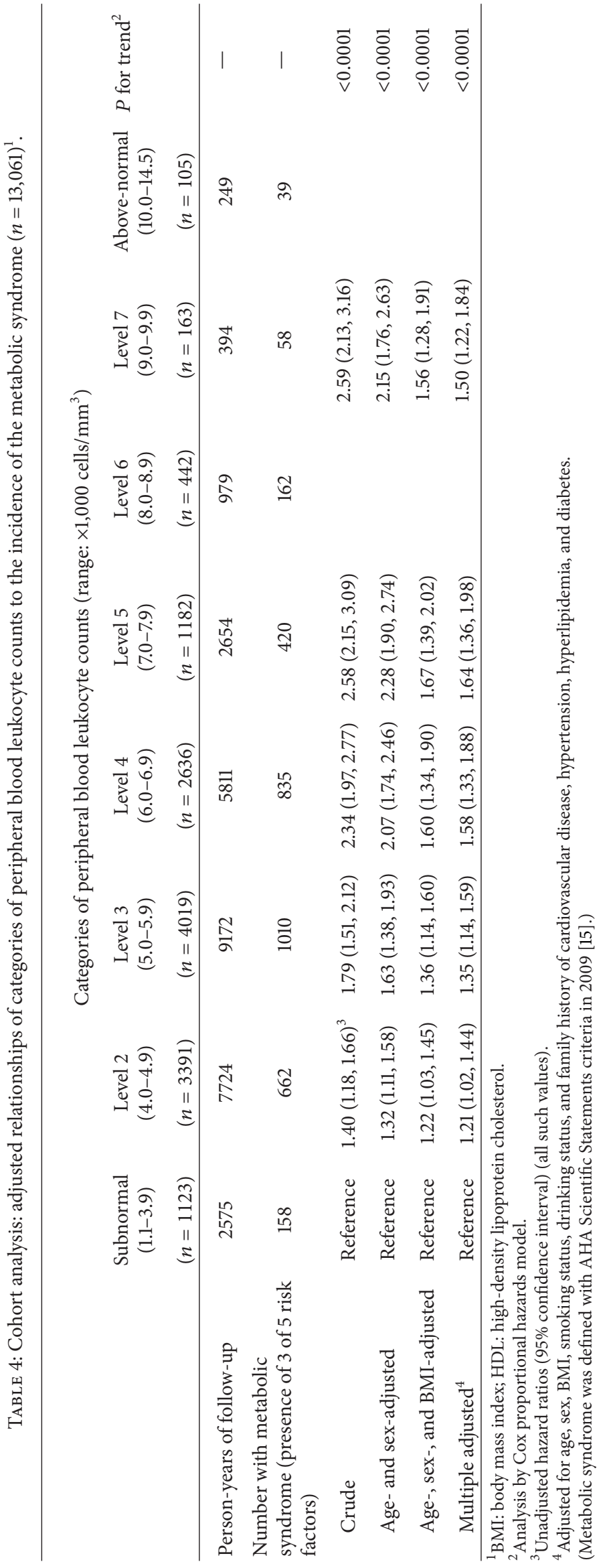


Cross-sectional analysis

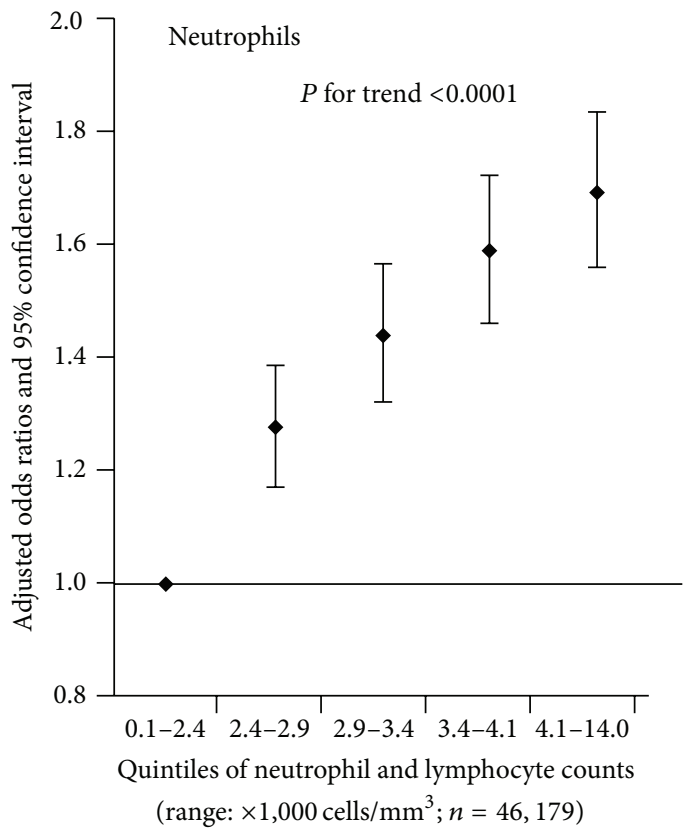

Cohort analysis

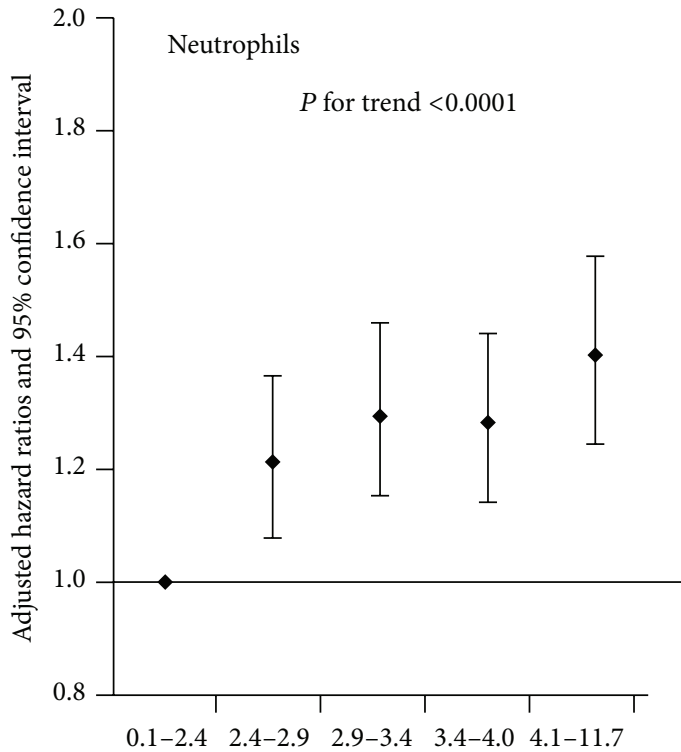

Quintiles of neutrophil and lymphocyte counts (range: $\times 1,000$ cells $/ \mathrm{mm}^{3} ; n=13,061$ )

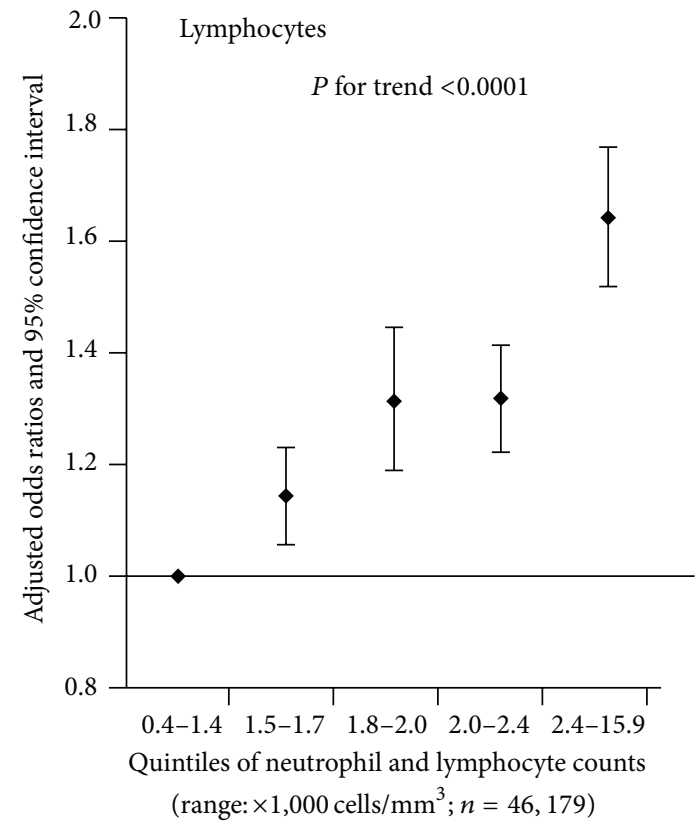

(a)

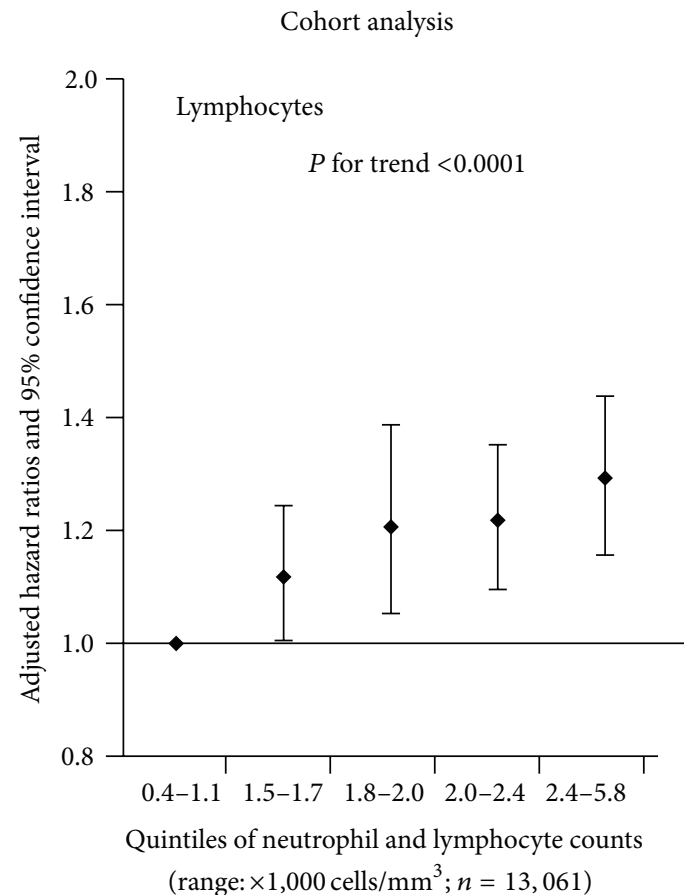

(b)

Figure 2: Adjusted odds ratios (a) and hazard ratios (b) (95\% confidence interval) of the relationship between the quintiles of neutrophil and lymphocyte counts and metabolic syndrome. Adjusted for age, sex, BMI, smoking status, drinking status, and family history of cardiovascular disease, hypertension, hyperlipidemia, and diabetes.

may potentially maintain better physical performance [26]. In the present study, we had hypothesized that the participants with subnormal leukocyte counts would have the healthiest states among apparently healthy adults. Thus, the subnormal category was used as a reference group in the analysis. In accordance with our hypothesis, the results show that the subnormal leukocyte count category had the lowest prevalence and incidence of MS. 
Leukocytes are part of the body's defense against foreign substances and infections. In clinical practice, the count of leukocytes is measured as part of a routine blood test and is an important way for doctors to gauge individual health. The reference range is generally established by a $95 \%$ lower and higher limit in the peripheral blood of a general population [5]. The conventional cutoff of 4,000 cells $/ \mathrm{mm}^{3}$ is considered a screening level for reduced leukocyte counts. However, in contrast to this general knowledge of clinical practice, we not only observed that subnormal leukocyte count levels were related to the lowest prevalence of MS in a crosssectional study, but also found a significant relationship to the lowest incidence of MS during a 5-year follow-up period among an apparently healthy population. These findings suggest that it is necessary to restudy and discuss the clinical or preventive value of subnormal leukocyte counts among normal populations.

Based on conventional recognition, Sakuragi and his colleagues reassessed the reference values for peripheral blood leukocyte counts among the general population and attempted to examine whether the reference range had changed in the past 100 years and what its impact factors were [5]. The study concluded that leukocyte counts had a secular trend of decrease in the past 100 years with decreased smoking rates and improved general hygiene being discussed as possible factors for this reduction. Indeed, the widespread use of antibiotics and improvement of general hygiene are considered important factors for reduction of inflammatory levels; however, a more important risk factor (obesity) was not discussed in the paper. Obesity causes many immune cells to infiltrate or populate in adipose tissue and promote chronic low-grade inflammation [27]. Furthermore, fat cells, particularly those in the visceral fat, are now considered an immune organ, secreting numerous immune modulating chemicals contributing directly to the development of lowgrade inflammation [28]. Over the past few decades, there has been a steep rise in the incidence of obesity worldwide. Thus, rather than general hygiene or bacterial infection, obesity has already become the strongest risk factor for a chronic inflammatory level $[29,30]$. In agreement with these studies, ours also suggests that the categories of leukocyte counts were strongly and significantly related to BMI even after adjustment for age and sex. More importantly, many studies have suggested that increases in inflammatory levels related to poor hygiene and obesity may be due to a different mechanism [29, 30]. Furthermore, mounting evidence highlights the role of adipose tissue in the development of a systemic inflammatory state that contributes to vasculopathy and cardiovascular risk. Therefore, we consider it necessary to clarify the exact mechanism and health effects of leukocyte count reduction over time and then reassess its clinical or preventive value among the general population.

Our results also showed that elevated neutrophil and lymphocyte counts were independently related to the prevalence and incidence of MS. Since neutrophils are the most abundant cell type involved in the innate immune response and the cells of the adaptive immune system are lymphocytes, the results suggest that the innate and adaptive immune responses may simultaneously increase the incidence of MS. Further studies are required to clarify the hypothesis.

Nutritional status is an important risk factor for decreased leukocyte counts [31]. However, the serum albumin level, a standard indicator of nutritional status, was not significantly different among the eight categories of leukocyte counts in our study population (see Tables 1 and 3). Therefore, we deduced that nutritional status is not a major reason for the lower leukocytes count in our study population.

The present study does have a limitation in that although we adjusted for a considerable number of potentially confounding factors, we cannot exclude the possibility that MS is affected by other lifestyle variables which are intrinsically related to leukocyte counts.

\section{Conclusions}

In conclusion, this large-scale epidemiological study has shown that subnormal leukocyte counts were independently related to the lowest prevalence and incidence of MS. Based on these findings, we have constructed the novel hypothesis that a subnormal leukocyte count is not a reference value for screening cases of reduced leukocyte counts, but is maybe the healthiest range for prevention and treatment of MS or CVD among an apparently healthy population. These findings also strongly suggest that it is necessary to restudy and discuss the clinical or preventive value and the possible mechanism of subnormal leukocyte counts among the general population.

\section{Conflict of Interests}

The authors declare that there is no conflict of interests regarding the publication of this paper.

\section{Authors' Contribution}

Shaomei Sun and Hongmei Wu are co-first authors with equal contribution.

\section{Acknowledgments}

The authors gratefully acknowledge all of the men and women who participated in the study and Tianjin Medical University General Hospital-Health Management Centre for creating the possibility to perform the study. This study was supported by grants from the key technologies R\&D program of Tianjin (Key Project nos. 11ZCGYSY05700, 12ZCZDSY20400, and 13ZCZDSY20200) and the Science Foundation of Tianjin Medical University (no. 2010KY28), China.

\section{References}

[1] R. Lozano, M. Naghavi, K. Foreman et al., "Global and regional mortality from 235 causes of death for 20 age groups in 1990 and 2010: a systematic analysis for the global burden of disease study 2010," The Lancet, vol. 380, no. 9859, pp. 2095-2128, 2012.

[2] T. A. Pearson, G. A. Mensah, R. W. Alexander et al., "Markers of inflammation and cardiovascular disease: application to clinical 
and public health practice: a statement for healthcare professionals from the centers for disease control and prevention and the American Heart Association," Circulation, vol. 107, no. 3, pp. 499-511, 2003.

[3] N. S. Jenny, "Inflammation in aging: cause, effect, or both?" Discovery Medicine, vol. 13, no. 73, pp. 451-460, 2012.

[4] D. W. Kamp, E. Shacter, and S. A. Weitzman, "Chronic inflammation and cancer: the role of the mitochondria," Oncology, vol. 25, no. 5, pp. 400-410, 413, 2011.

[5] S. Sakuragi, J. Moriguchi, F. Ohashi, and M. Ikeda, "Reference value and annual trend of white blood cell counts among adult Japanese population," Environmental Health and Preventive Medicine, vol. 18, no. 2, pp. 143-150, 2013.

[6] E. Oda and R. Kawai, "The prevalence of metabolic syndrome and diabetes increases through the quartiles of white blood cell count in Japanese men and women," Internal Medicine, vol. 48, no. 13, pp. 1127-1134, 2009.

[7] D.-J. Kim, J.-H. Noh, B.-W. Lee et al., "The associations of total and differential white blood cell counts with obesity, hypertension, dyslipidemia and glucose intolerance in a Korean population," Journal of Korean Medical Science, vol. 23, no. 2, pp. 193-198, 2008.

[8] Y.-Y. Wang, S.-Y. Lin, P.-H. Liu, B. M. H. Cheung, and W.-A. Lai, "Association between hematological parameters and metabolic syndrome components in a Chinese population," Journal of Diabetes and its Complications, vol. 18, no. 6, pp. 322-327, 2004.

[9] K. Odagiri, A. Uehara, I. Mizuta, M. Yamamoto, and C. Kurata, "Longitudinal study on white blood cell count and the incidence of metabolic syndrome," Internal Medicine, vol. 50, no. 21, pp. 2491-2498, 2011.

[10] W. Meng, C. Zhang, Q. Zhang et al., "Association between leukocyte and metabolic syndrome in urban Han Chinese: a longitudinal cohort study," PLoS ONE, vol. 7, no. 11, Article ID e49875, 2012.

[11] N. Babio, N. Ibarrola-Jurado, M. Bullo et al., "White blood cell counts as risk markers of developing metabolic syndrome and its components in the Predimed study," PLoS ONE, vol. 8, no. 3, Article ID e58354, 2013.

[12] K. Tamakoshi, H. Toyoshima, H. Yatsuya et al., "White blood cell count and risk of all-cause and cardiovascular mortality in nationwide sample of Japanese-results from the NIPPON DATA90," Circulation Journal, vol. 71, no. 4, pp. 479-485, 2007.

[13] K. Song, H. Du, Q. Zhang et al., "Serum immunoglobulin $\mathrm{M}$ concentration is positively related to metabolic syndrome in an adult population: tianjin chronic low-grade systemic inflammation and health (TCLSIH) cohort study," PLOS ONE, vol. 9, no. 2, Article ID e88701, 2014.

[14] “About Tianjin,” 2013http://www.tj.gov.cn/english/About_tianjin/Visiting_Tianjin/.

[15] G. Maresca, A. di Blasio, R. Marchioli, and G. di Minno, "Measuring plasma fibrinogen to predict stroke and myocardial infarction: an update," Arteriosclerosis, Thrombosis, and Vascular Biology, vol. 19, no. 6, pp. 1368-1377, 1999.

[16] K. G. M. M. Alberti, R. H. Eckel, S. M. Grundy et al., "Harmonizing the metabolic syndrome: a joint interim statement of the international diabetes federation task force on epidemiology and prevention; national heart, lung, and blood institute; American heart association; world heart federation; international atherosclerosis society; and international association for the study of obesity," Circulation, vol. 120, no. 16, pp. 1640-1645, 2009.
[17] H. Y. Chung, M. Cesari, S. Anton et al., "Molecular inflammation: underpinnings of aging and age-related diseases," Ageing Research Reviews, vol. 8, no. 1, pp. 18-30, 2009.

[18] C. E. Lewis, K. M. McTigue, L. E. Burke et al., "Mortality, health outcomes, and body mass index in the overweight range: a science advisory from the american heart association," Circulation, vol. 119, no. 25, pp. 3263-3271, 2009.

[19] F. Corre, J. Lellouch, and D. Schwartz, "Smoking and leucocytecounts. Results of an epidemiological survey," The Lancet, vol. 2, no. 7725, pp. 632-634, 1971.

[20] J. B. Zalokar, J. L. Richard, and J. R. Claude, "Leukocyte count, smoking, and myocardial infarction," The New England Journal of Medicine, vol. 304, no. 8, pp. 465-468, 1981.

[21] E. Oda and R. Kawai, "Comparison between high-sensitivity Creactive protein (hs-CRP) and white blood cell count (WBC) as an inflammatory component of metabolic syndrome in Japanese," Internal Medicine, vol. 49, no. 2, pp. 117-124, 2010.

[22] Y. H. Kang, H. G. Min, I. J. Kim, Y. K. Kim, and S. M. Son, "Comparison of alanine aminotransferase, white blood cell count, and uric acid in their association with metabolic syndrome: a study of Korean adults," Endocrine Journal, vol. 55, no. 6, pp. 1093-1102, 2008.

[23] G. P. Fadini, G. Marcuzzo, M. C. Marescotti, S. V. de Kreutzenbergand, and A. Avogaro, "Elevated white blood cell count is associated with prevalence and development of the metabolic syndrome and its components in the general population," Acta Diabetologica, vol. 49, no. 6, pp. 445-451, 2012.

[24] N. Nakanishi, K. Suzuki, and K. Tatara, "White blood cell count and clustered features of metabolic syndrome in Japanese male office workers," Occupational Medicine, vol. 52, no. 4, pp. 213$218,2002$.

[25] K. I. Kim, J. Lee, N. J. Heo et al., "Differential white blood cell count and all-cause mortality in the Korean elderly," Experimental Gerontology, vol. 48, no. 2, pp. 103-108, 2013.

[26] K. Niu, A. Hozawa, H. Guo et al., "Serum C-reactive protein even at very low $(<1.0 \mathrm{mg} / \mathrm{l})$ concentration is associated with physical performance in a community-based elderly population aged 70 years and over," Gerontology, vol. 54, no. 5, pp. 260-267, 2008.

[27] S. Sun, Y. Ji, S. Kersten, and L. Qi, "Mechanisms of inflammatory responses in obese adipose tissue," Annual Review of Nutrition, vol. 32, pp. 261-286, 2012.

[28] H. Sell and J. Eckel, "Adipose tissue inflammation: novel insight into the role of macrophages and lymphocytes," Current Opinion in Clinical Nutrition and Metabolic Care, vol. 13, no. 4, pp. 366-370, 2010.

[29] C. Nathan, "Epidemic inflammation: pondering obesity," Molecular Medicine, vol. 14, no. 7-8, pp. 485-492, 2008.

[30] P. Calabrò, E. Golia, V. Maddaloni et al., "Adipose tissuemediated inflammation: the missing link between obesity and cardiovascular disease?" Internal and Emergency Medicine, vol. 4, no. 1, pp. 25-34, 2009.

[31] W. Y. Hsu, C. H. Wu, C. T. Hsieh, H. C. Lo, J. S. Lin, and M. D. Kao, "Low body weight gain, low white blood cell count and high serum ferritin as markers of poor nutrition and increased risk for preterm delivery," Asia Pacific Journal of Clinical Nutrition, vol. 22, no. 1, pp. 90-99, 2013. 


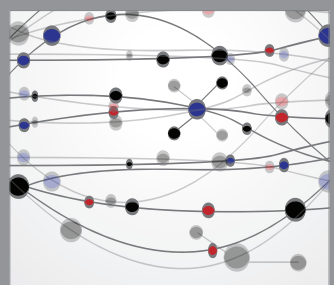

The Scientific World Journal
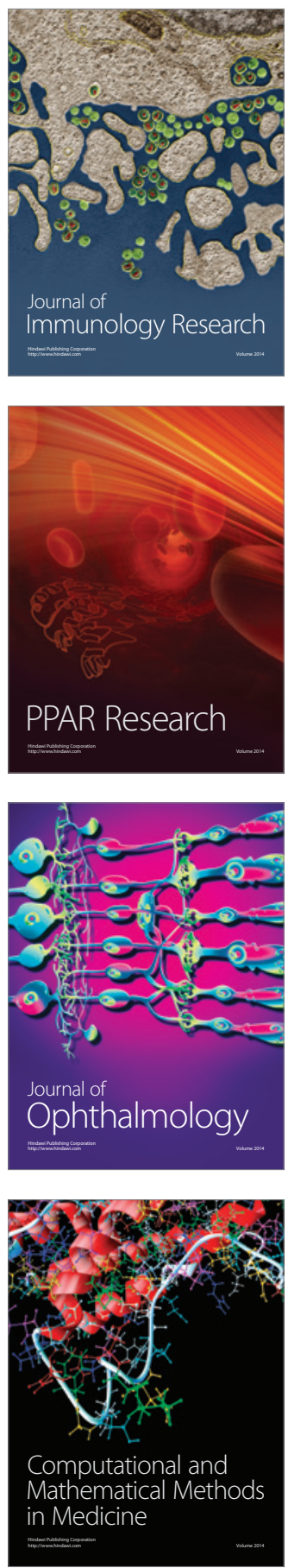

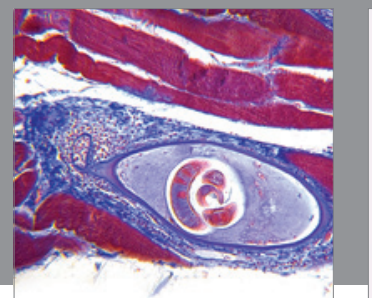

Gastroenterology

Research and Practice
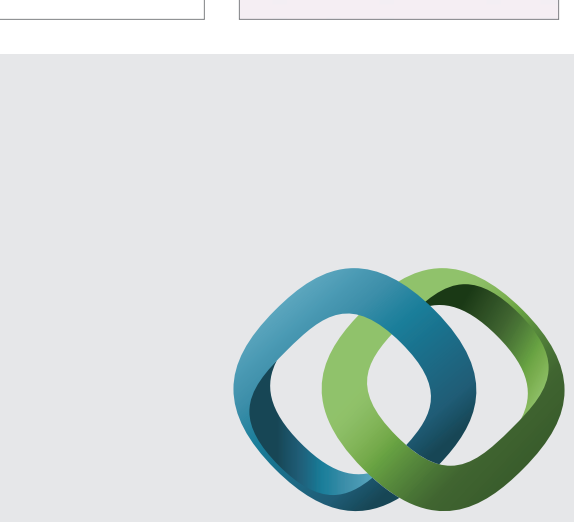

\section{Hindawi}

Submit your manuscripts at

http://www.hindawi.com
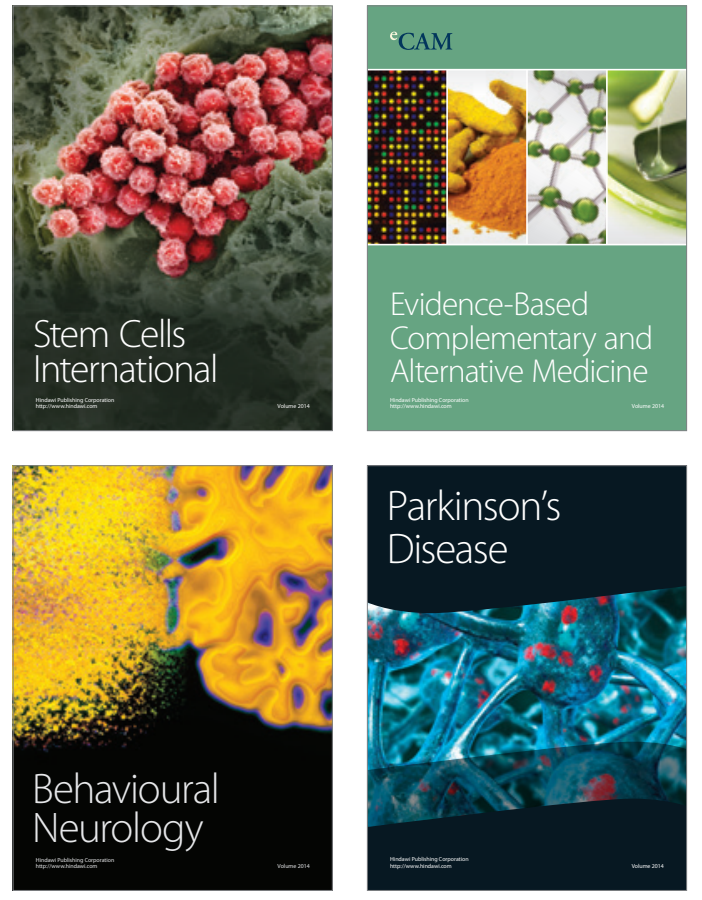
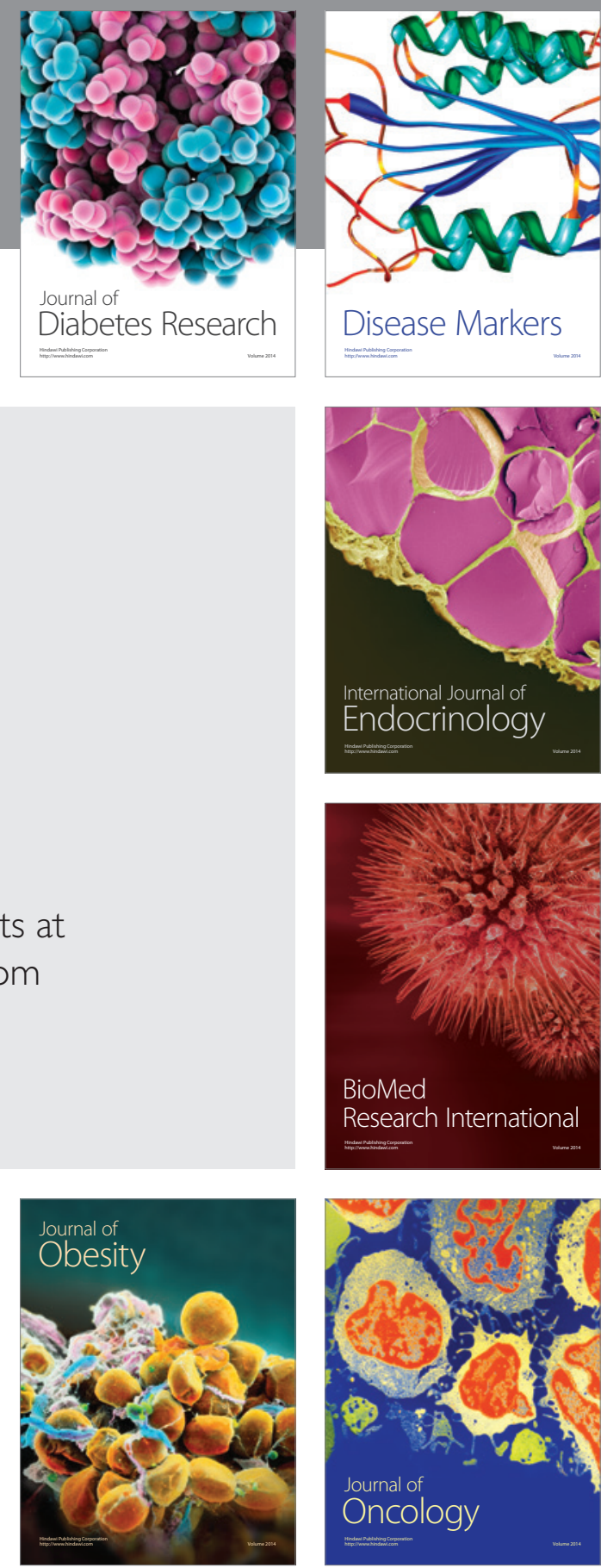

Disease Markers
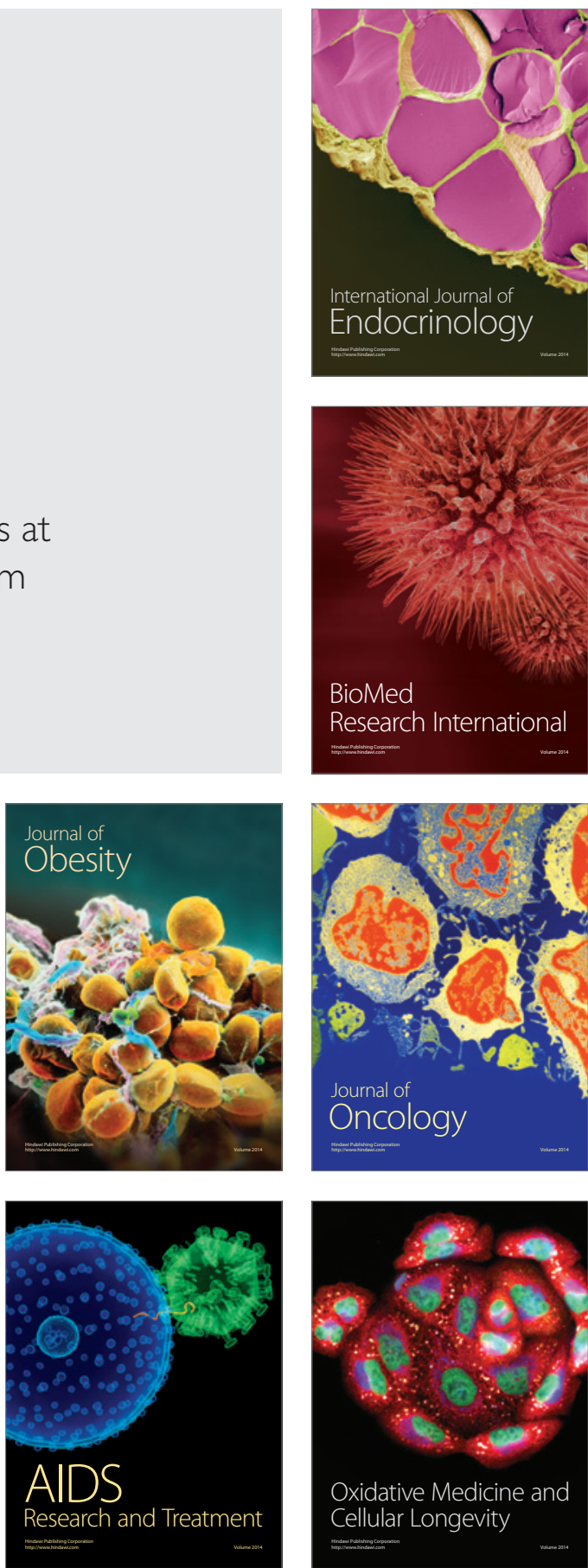